PAReIGIS, $\mathrm{B}$.

Math. Zeitschr. 93, 265-275 (1966)

\title{
Vergessende Funktoren und Ringhomomorphismen
}

\author{
BODO PAREIGIS
}

Eingegangen am 25. März 1966

Ein ,vergessender“ Funktor (engl. ,forgetful“) folgender Art wird betrachtet. Seien $R, S$ unitäre, assoziative Ringe und sei $f: S \rightarrow R$ ein unitärer Ringhomomorphismus. Jedem unitären $R$-Links-Modul ${ }_{R} M$ wird der folgende unitäre $S$-Links-Modul ${ }_{S} M$ zugeordnet: die unterliegende abelsche Gruppe sei $M$, die auch die zu ${ }_{R} M$ gehörige abelsche Gruppe ist, und die Operation von $S$ auf $M$ werde definiert durch $s \cdot m:=f(s) m$ für $s \in S$ und $m \in M$. Jeder $R$-Homomorphismus von $R$-Moduln ist dann auch ein $S$-Homomorphismus bezüglich der beschriebenen $S$-Struktur. Durch den Homomorphismus $f: S \rightarrow R$ wird auf diese Weise ein Funktor $\Omega(f)$ von der Kategorie der unitären $R$-Links-Moduln ${ }_{R} \mathfrak{H b}$ in die Kategorie der unitären $S$-Links-Moduln ${ }_{s}^{\mathfrak{I} b}$ induziert. Dieser Funktor ,vergißt“" die $R$-Struktur von ${ }_{R} M$ und erhält nur die vermöge $f$ definierte $S$-Struktur. Die ,vergessenden“ Funktoren treten oft in der relativen Homologietheorie auf (z.B. in MacLane: Homology).

MoRITA [5] verwendet gewisse vergessende Funktoren zur Charakterisierung von Frobeniuserweiterungen. Er entwickelt damit einen großen Teil der bisher bekannten Theorie der Frobeniuserweiterungen.

Wir wollen in dieser Arbeit einige grundlegende Tatsachen über die Theorie der vergessenden Funktoren entwickeln, die sofort erkennen lassen, daß die vergessenden Funktoren alle kategorietheoretischen Eigenschaften einer Ringerweiterung enthalten und daher geeignete Objekte zum Studium von Ringerweiterungen sind.

Mit dieser Auffassung ergeben sich als naturgemäße Folgerungen der Theorie einige bekannte Sätze aus der Ringtheorie, wie etwa die Sätze über den Endomorphismenring einer Frobeniuserweiterung (Folgerung 5.3).

\section{Bezeichnungen und mengentheoretische Voraussetzungen}

Wir nehmen an, daß zu jeder Menge $M$ ein Grothendieck-Universum $\mathfrak{U}$ mit $M \in \mathfrak{H}$ existiert [2]. Sei ein festes Universum $\mathfrak{U}$ so gewählt, daß alle Mengen, auf die die folgenden Sätze angewendet werden sollen, schon aus $\mathfrak{U}$ sind. Sei weiterhin $\mathfrak{U} \in \mathfrak{B}$ und $\mathfrak{B} \in \mathfrak{M}$ für weitere Universen $\mathfrak{B}$ und $\mathfrak{M}$.

Sei $A$ ein fest gewählter kommutativer, assoziativer Ring mit Einselement mit $A \in \mathfrak{U}$. Die vorkommenden Modulkategorien und $A$-präadditiven Kategorien seien U-Kategorien (i. S.v. [2], S. 330) und die Menge der Objekte einer solchen Kategorie gehöre zu $\mathfrak{B}$.

19 Math. Z., Bd. 93 
Wir definieren jetzt:

$\mathscr{A}$ sei die Kategorie der assoziativen $A$-Algebren mit Einselement und der unitären $A$-Algebrenhomomorphismen. Wenn die unterliegenden Mengen $\operatorname{der} A$-Algebren in $\mathfrak{U}$ bzw. $\mathfrak{B}$ liegen und die Menge der Objekte von $\mathscr{A}$ in $\mathfrak{U}$ bzw. $\mathfrak{B}$ enthalten ist, schreiben wir oft auch $\mathfrak{U} \mathscr{A}$ bzw. $\mathfrak{B} \mathscr{A}$.

$\mathscr{P}$ sei die Kategorie der $A$-präadditiven Kategorien mit Isomorphieklassen von $A$-additiven Funktoren als Morphismen. Nach der Voraussetzung über $A$-präadditive Kategorien ist $\mathscr{P}$ eine $\mathfrak{B}$-Kategorie. Wir wollen die Funktoren und ihre Isomorphieklassen in $\mathscr{P}$ mit denselben Buchstaben bezeichnen.

${ }^{\mathfrak{r}} \mathscr{P}$ mit einer $A$-präadditiven Kategorie $\mathfrak{A}$ aus $\mathscr{P}$ sei die Kategorie $\operatorname{der} A$-präadditiven Kategorien über $\mathfrak{A}$, d.h. der Paare $(\mathfrak{B}, F)$ bestehend aus einer $A$-präadditiven Kategorie $\mathfrak{B}$ und einer Isomorphieklasse von $A$-additiven Funktoren $F: \mathfrak{B} \rightarrow \mathfrak{U}$. Die Morphismen von $(\mathfrak{B}, F)$ nach $(\mathfrak{C}, G)$ seien Isomorphieklassen von $A$-additiven Funktoren $H: \mathfrak{B} \rightarrow \mathfrak{C}$ über $\mathfrak{A}$, d.h. so daß $F=G H$.

$\mathscr{M}$ en sei die Kategorie aller Mengen, die Element von $\mathfrak{B}$ sind, und aller Mengenabbildungen.

$\mathscr{A} \mathscr{M} e n$ sei die Kategorie der kovarianten Funktoren von $\mathscr{A}$ in $\mathscr{M} e n$. Wenn $\mathscr{A}=\mathfrak{u} \mathscr{A}$ bzw. $\mathfrak{B} \mathscr{A}$ ist, dann ist $\mathscr{A} \mathscr{M}$ en eine $\mathfrak{B}$ - bzw. $\mathfrak{W}$-Kategorie.

\section{Einbettungen von $\mathscr{A}$ in $\mathscr{P}$}

Sei $A$ ein kommutativer Ring und $\mathfrak{A}$ ein Objekt aus $\mathscr{P}$. Eine $A$-Algebra $R$ aus $\mathfrak{B} \mathscr{A}$ fassen wir auf als eine $A$-präadditive Kategorie mit einem Objekt (mit $R$ bezeichnet) und Endomorphismenring $R$. Wir definieren $\operatorname{Funkt}_{A}(R, \mathfrak{U})$ als die Kategorie der $A$-additiven Funktoren von $R$ in $\mathfrak{A}$. Wegen der Kommutativität von $A$ ist diese wieder eine $A$-präadditive Kategorie in $\mathscr{P}$.

Ist $\mathfrak{A}={ }_{B} \mathfrak{A} \mathfrak{b}$ die Kategorie der unitären $B$-Moduln für eine $A$-Algebra $B$, so ist $\mathrm{Funkt}_{A}\left(R,{ }_{B} \mathfrak{A} \mathfrak{b}\right) \cong{ }_{S} \mathfrak{O b}$ mit $S \cong R \otimes_{A} B$.

Von $F_{u n k t}(R, \mathfrak{A})$ in $\mathfrak{A}$ haben wir in natürlicher Weise einen Funktor $V$, der jedem Funktor $F \in \mathrm{Funkt}_{A}(R, \mathfrak{A})$ das Objekt $F(R)$ aus $\mathfrak{U}$ zuordnet. Dieser Funktor ist $A$-additiv. $A$-Algebrenhomomorphismen $f: R \rightarrow S$ bzw. $A$-additive Funktoren $G: \mathfrak{A} \rightarrow \mathfrak{B}$ induzieren Funktoren $\tilde{f}: F \operatorname{Funt}_{A}(S, \mathfrak{A}) \rightarrow \operatorname{Funkt}_{A}(R, \mathfrak{A})$ bzw. $\widetilde{G}:$ Funkt $_{A}(R, \mathfrak{U}) \rightarrow$ Funkt $_{A}(R, \mathfrak{B})$. Bezüglich dieser Funktoren sind die Funktoren $V$ natürlich, d.h. sie kommutieren mit ihnen.

Wir haben damit einen Funktor $\Omega: \mathscr{A}^{0} \rightarrow^{\mathscr{2} \mathscr{P}}$ definiert, wobei $\mathscr{A}^{0}$ die zu $\mathscr{A}$ duale Kategorie sei, nämlich $\Omega(R)=\left(\mathrm{Funkt}_{A}(R, \mathfrak{U}), V\right)$ und $\Omega(f)=$ $\tilde{f}: \operatorname{Funkt}_{A}(S, \mathfrak{O}) \rightarrow \operatorname{Funkt}_{A}(R, \mathfrak{O})$.

Bei Anwendungen wird im wesentlichen der Spezialfall $A=\boldsymbol{Z}$ und $\mathfrak{A}=\mathfrak{A} \mathfrak{b}$ interessieren, d.h. dann ist $\Omega: \mathscr{A}^{0} \rightarrow^{\mathfrak{M b}} \mathscr{P}$ so definiert, daß jedem Ring die Kategorie der Moduln über diesem Ring zugeordnet wird zusammen mit dem Funktor, der jedem Modul die unterliegende abelsche Gruppe zuordnet.

Wieder in etwas größerer Allgemeinheit definieren wir einen Funktor $\Gamma:{ }^{\mathfrak{A} P} \rightarrow \mathscr{A}^{0}$ in der folgenden Weise: Sei $(\mathfrak{B}, F)$ ein Objekt aus ${ }^{\mathfrak{A}} \mathscr{P}$. Dann definieren wir $\Gamma(\mathfrak{B}, F)=$ End $(F)$, den Endomorphismenring von $F$. End $(F)$ ist eine $A$-Algebra, also ein Objekt in $\mathscr{A}$. Sei jetzt $G:(\mathfrak{B}, F) \rightarrow(\mathfrak{C}, H)$ ein Mor- 
phismus in $\mathscr{\mathscr { P }}$, das Diagramm<smiles>[B]1CCCC1</smiles>

sei bis auf Isomorphie von Funktoren kommutativ: $H G \cong F$. Dann existieren $A$-Algebrenhomomorphismen $\operatorname{End}(H) \stackrel{G}{\rightarrow} \operatorname{End}(H G) \cong \operatorname{End}(F)$. Die Zusammensetzung bezeichnen wir mit $\Gamma(G)$.

Im Spezialfall $A=\boldsymbol{Z}$ und $\mathfrak{A}=\mathfrak{A} \mathfrak{b}$ haben wir damit einen Funktor $\Gamma:{ }^{\mathscr{T} 6} \mathscr{P} \rightarrow \mathscr{A}^{0}$ konstruiert, wobei $\mathscr{A}$ die Kategorie der Ringe ist.

Satz 1.1. $\Gamma$ ist links-adjungierter Funktor zu $\Omega$, d.h. es existiert ein natürlicher Isomorphismus

$$
\mathscr{P}_{\mathscr{P}}(\mathfrak{B}, \Omega R) \cong \mathscr{A}^{0}(\Gamma \mathfrak{B}, R) .
$$

Beweis. Wir wollen nur die jeweiligen Zuordnungen angeben. Sei zunächst $F: \mathfrak{B} \rightarrow \Omega R$ ein Funktor, dessen Isomorphieklasse in $\mathscr{P}$ liege. Dann haben wir ein Diagramm

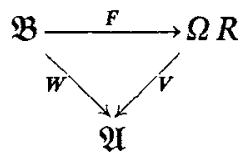

mit $\alpha: V F \cong W$, wobei alle Funktoren $A$-additiv sind. Wir ordnen dem Funktor $F$ einen $A$-Algebrenhomomorphismus $f^{\prime}: R \rightarrow \operatorname{End}(W)$ zu vermöge des kommutativen Diagramms

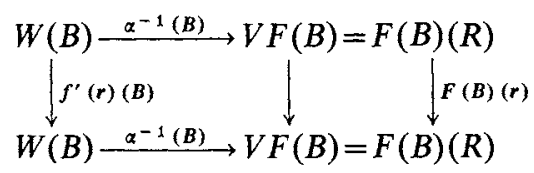

d. h. wir definieren $f^{\prime}(r)(B)=\alpha(B) \cdot F(B)(r) \cdot \alpha^{-1}(B)$.

Sei nun umgekehrt ein $A$-Algebrenhomomorphismus $f: R \rightarrow \operatorname{End}(W)$ gegeben. Dann definieren wir einen Funktor $F^{\prime}: \mathfrak{B} \rightarrow \Omega R$ durch

$$
\begin{aligned}
& F^{\prime}(B)(R):=W(B) \\
& F^{\prime}(B)(r):=f(r)(B) \\
& F^{\prime}(\beta)(R):=W(\beta) .
\end{aligned}
$$

Man sieht sofort, daß die Isomorphieklasse von $F^{\prime}$ einen Morphismus in 2\% $\mathscr{P P}$ definiert.

Die Zuordnung $f \sim F^{\prime} \sim f^{\prime \prime}$ ist die Identität, d.h. $f^{\prime \prime}=f$, und die Zuordnung $F \sim f^{\prime} \leadsto F^{\prime \prime}$ hat die Eigenschaft, daß $\tilde{\alpha}: F \cong F^{\prime \prime}$, wobei $\tilde{\alpha}(B)(R):=\alpha(B)$. Also definieren $F$ und $F^{\prime \prime}$ denselben Morphismus in $\mathscr{P} \mathscr{P}$. Weiter rechnet man leicht nach, daß diese Zuordnungen funktoriell in $\mathfrak{B}$ bzw. $R$ sind.

19* 
Satz 1.2. Sei $\mathfrak{U}={ }_{A} \mathfrak{A} \mathfrak{b}$, die Kategorie der unitären A-Moduln. Dann ist $\Omega: \mathscr{A}^{0} \rightarrow^{\mathfrak{A}} \mathscr{P}$ eine volle Einbettung.

Lemma 1.3. Die natürliche Transformation $\operatorname{Id}\left(\mathscr{A}^{0}\right) \rightarrow \Gamma \Omega$ ist ein Isomorphismus.

Beweis. Wir berechnen explizit den Funktor $V: \Omega R \rightarrow{ }_{A} \mathfrak{U b}$. Er ist $V \cong \operatorname{Hom}_{R}(R, \cdot)$. Also ist der Endomorphismenring $\operatorname{End}(V) \cong \operatorname{End}\left(\operatorname{Hom}_{R}(R, \cdot)\right)$ $\cong \operatorname{End}_{R}(R)^{\circ} \cong R$, und dieser Isomorphismus definiert die natürliche Transformation $\operatorname{Id}\left(\mathscr{A}^{0}\right) \rightarrow \Gamma \Omega$, ist also funktoriell.

Beweis des Satzes. Wir haben zu zeigen, daß $\Omega: \mathscr{A}^{\circ}(R, S) \rightarrow^{\mathfrak{A} \mathscr{P}}(\Omega R, \Omega S)$ ein Isomorphismus ist. Aber das Diagramm

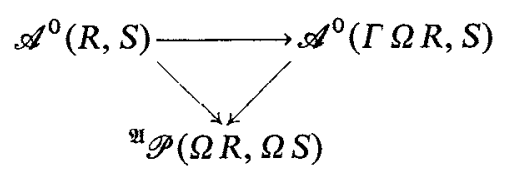

ist kommutativ, und alle Abbildungen sind Isomorphismen.

Folgerung 1.4. $\lim _{\longleftarrow} \cong \Omega \lim , \underline{\lim } \Gamma \cong \Gamma \underline{\lim }$.

Folgerung 1.5. In ${ }^{16} \mathscr{P}$ existiert der projektive Limes von Modulkategorien $\left({ }_{R} \mathfrak{U b}, V:{ }_{R} \mathfrak{U b} \rightarrow \mathfrak{U} \mathfrak{b}\right)$ und ist wieder eine Modulkategorie.

Satz 1.6. In $\mathscr{\Re}$ existieren induktive Limites.

Beweis. Es genügt zu zeigen, daß in $\mathscr{P} \mathscr{P}$ direkte Summen und Kokerne existieren. Sei $\left\{\mathfrak{B}_{\boldsymbol{i}}\right\}_{i \in I}$ eine Familie von $A$-präadditiven Kategorien über $\mathfrak{A}$. Wir definieren eine neue $A$-präadditive Kategorie $\coprod \mathfrak{B}_{i}$ über $\mathfrak{A}$ durch

$$
\begin{aligned}
\operatorname{Obj} \amalg \mathfrak{B}_{i}= & \bigcup \operatorname{Obj}_{\mathfrak{B}_{i}} \\
\operatorname{Mor} \amalg \mathfrak{B}_{i}= & \left(\bigcup \operatorname{Mor}_{i} \mathfrak{B}_{i}\right) \cup\left\{\operatorname{Mor}\left(B_{i p}, B_{j}\right)=0 \text { für } B_{i} \in \operatorname{Obj}_{i},\right. \\
& \left.B_{j} \in \operatorname{Obj}_{j} \text { und } i \neq j\right\} .
\end{aligned}
$$

Der Funktor von $\llbracket \mathfrak{B}_{i}$ in $\mathfrak{A}$ ist elementweise durch die Funktoren der $\mathfrak{B}_{\boldsymbol{i}}$ in $\mathfrak{A}$ definiert.

Seien $F, G: \mathfrak{B} \rightarrow \mathfrak{C}$ zwei $A$-additive Funktoren in $\mathscr{M} \mathscr{P}$. Wir definieren eine $A$-präadditive Kategorie $\operatorname{Kok}(F, G)$ über $\mathfrak{U}$ und einen $A$-additiven Funktor $H: \mathfrak{C} \rightarrow \operatorname{Kok}(F, G)$, die zusammen einen Kokern von $F$ und $G$ repräsentieren. Wir setzen

$$
\begin{aligned}
\operatorname{Obj} \operatorname{Kok}(F, G)= & \operatorname{Obj} \mathbb{C} \\
\operatorname{Mor} \operatorname{Kok}(F, G)= & \left\langle\operatorname{Mor} \mathfrak{C}, i_{B}: F(B) \rightarrow G(B), j_{B}: G(B) \rightarrow F(B)\right. \\
& \text { für alle } B \in \operatorname{Obj} \mathfrak{B} \mid i_{B} \cdot j_{B}=\operatorname{id}(G(B)), \\
& j_{B} \cdot i_{B}=\operatorname{id}(F(B)), G(\psi) \cdot i_{B}=i_{B^{\prime}} \cdot F(\psi)
\end{aligned}
$$

für alle $\left.\psi \in \operatorname{Hom}\left(B, B^{\prime}\right)\right\rangle$. 
Dabei sei \langle\rangle die durch Summen, Multiplikation mit Elementen aus $A$ und Verknüpfung von Morphismen erzeugte Morphismenmenge, wobei die angegebenen Relationen entsprechend berücksichtigt werden müssen. Weiter definieren wir $H(B)=B, H(\varphi)=\varphi$ modulo der angegebenen Relationen in Mor $\operatorname{Kok}(F, G)$. $(\operatorname{Kok}(F, G), H)$ ist ein Kokern in $\mathscr{P}$, also existiert ein Funktor von $\operatorname{Kok}(F, G)$ in $\mathfrak{H}$, so daß $H$ über $\mathfrak{A}$ steht. Damit ist $(\operatorname{Kok}(F, G), H)$ auch ein Kokern in ${ }^{\mathfrak{A}} \mathscr{P}$.

\section{Der Satz von Kan}

Wir wollen einen Funktor $\Theta$ von $\mathfrak{H} \mathscr{A}^{0}$ in $\mathscr{A} \mathscr{M} e n$ definieren durch $\Theta(R)=$ $\mathscr{A}(R, \cdot)$ und $\Theta(f)=\mathscr{A}(f, \cdot)$.

Satz 2.1. $\Theta: \mathscr{A}^{0} \rightarrow \mathscr{A} \mathscr{M}$ en ist eine volle Einbettung.

Der Beweis folgt aus [2, S. 332, Cor. 1].

Satz 2.2. Sei $A$ ein kommutativer Ring und $\mathfrak{A}={ }_{A} \mathfrak{H b}$. Dann existieren Funktoren $\Phi: \mathscr{A} \mathscr{M}$ en $\rightarrow{ }^{\mathscr{M} P}$ und $\Psi:{ }^{\mathfrak{Y}} \mathscr{P} \rightarrow \mathscr{A} \mathscr{M}$ en, die durch folgende Eigenschaften bis auf Isomorphie eindeutig gekennzeichnet sind:

1) $\Phi \Theta$ ist isomorph zu $\Omega$.

2) $\Phi$ ist links-adjungiert $z u \Psi$.

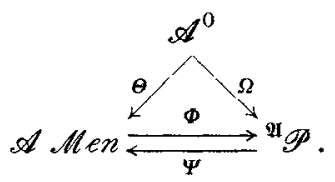

Beweis. Der Beweis verläuft im Prinzip wie in $[6$, S. 10]. $\Phi$ und $\Psi$ sind definiert durch

$$
\begin{aligned}
\Phi(\mathfrak{F}) & =\lim \Omega(S) \quad \text { für alle } \Theta(S) \text { über } \mathfrak{F} \\
\Psi(\mathfrak{B})(R) & ={ }^{\mathscr{H}} \mathscr{P}(\Omega R, \mathfrak{B}) .
\end{aligned}
$$

Der Aufbau der Theorie bisher enthält viele Parallelen zum Aufbau der algebraischen Geometrie (etwa in [6]). Es ist eine offene Frage, ob im kommutativen Fall, d.h. wenn ${ }^{\mathfrak{A}} \mathscr{P}$ nur aus denjenigen $A$-präadditiven Kategorien $\mathfrak{B}$ besteht, für die $\Gamma \mathfrak{B}$ eine kommutative $A$-Algebra ist, ${ }^{\mathfrak{a}} \mathscr{P}$ irgendeine Beziehung zur Kategorie der geometrischen Räume hat.

\section{Vergessende Funktoren}

Bei der Konstruktion von $\Omega: \mathscr{A}^{0} \rightarrow{ }^{\mathfrak{H} \mathscr{P}}$ traten Funktoren $V: F_{\text {funkt }}(R, \mathfrak{U}) \rightarrow \mathfrak{A}$ auf, die wir jetzt für den Fall $\mathfrak{U}={ }_{B} \mathfrak{A b}$ genauer untersuchen wollen. $B$ sei dabei eine $A$-Algebra. Wir deuten $F_{\text {unkt }}\left(R,{ }_{B} \mathfrak{A b}\right)$ als ${ }_{S} \mathfrak{A b}$ mit $S=R \otimes_{A} B . V$ induziert einen Funktor von $s^{\mathfrak{M b}}$ in ${ }_{B} \mathfrak{A b}$, den wir wieder mit $V$ bezeichnen wollen. $V$ ordnet jedem $S$-Modul den unterliegenden vermöge $B \rightarrow R \otimes_{A} B$ definierten $B$-Modul zu. Wir haben aber schon einen allgemeineren Fall dieses Funktors $V$ kennengelernt. Sei nämlich $f: R \rightarrow S$ ein $A$-Algebrenhomomorphismus und sei $\mathfrak{U}={ }_{A} \mathfrak{A b}$, so definiert $\Omega(f)$ einen $A$-additiven Funktor von $s^{\mathfrak{U b}}$ in ${ }_{R} \mathfrak{A b}$, der jedem $S$-Modul den unterliegenden vermöge $f: R \rightarrow S$ definierten $R$-Modul 
zuordnet. Diese ,,vergessenden“ Funktoren werden wir jetzt durch ihre Eigenschaften charakterisieren.

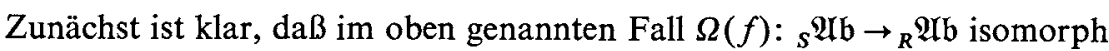

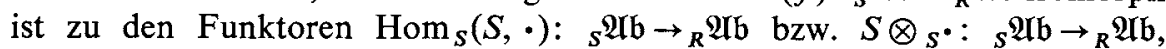
wobei $\operatorname{Hom}_{S}(S, M)$ bzw. $S \otimes_{S} M$ zu $R$-Moduln werden vermöge der $R$-Struktur auf $S$, die mit $f: R \rightarrow S$ gegeben ist. Der Funktor $\operatorname{Hom}_{S}(S, \cdot)$ hat aber einen links-adjungierten Funktor $S \otimes_{R} \cdot:{ }_{R} \mathfrak{A b} \rightarrow{ }_{S} \mathfrak{N l b}$ und der Funktor $S \otimes_{S} \cdot$ einen rechts-adjungierten Funktor $\operatorname{Hom}_{R}(S, \cdot):{ }_{R} \mathfrak{l} \mathfrak{l b} \rightarrow{ }_{s} \mathfrak{N} \mathfrak{l b}$, die wir auch mit * $\Omega(f)$ bzw. $\Omega(f)^{*}$ bezeichnen wollen. Weiter ist klar, daß bei dem Funktor $\Omega(f)$ die Homomorphismenmengen injektiv abgebildet werden. Wir geben daher folgende

Definition. Ein Funktor $F$ in $\mathscr{P}$ heißt vergessender Funktor, wenn $F$ eine Einbettung ist und wenn rechts- und links-adjungierte Funktoren zu $F$ in $\mathscr{P}$ existieren.

Diese Definition erhält ihre Berechtigung durch den

Satz 3.1. Sei $R$ eine A-Algebra und $\mathfrak{B}$ eine abelsche Kategorie in $\mathscr{P}$. Ein A-additiver Funktor $F: \mathfrak{B} \rightarrow{ }_{R} \mathfrak{A b}$ ist genau dann ein vergessender Funktor, wenn es eine A-Algebra $S$ und einen A-Algebrenhomomorphismus $f: R \rightarrow S$ so gibt, daß $s^{\mathfrak{U} \mathfrak{b}}$ zu $\mathfrak{B}$ äquivalent ist und der durch $F$ auf $s^{\mathfrak{A} \mathfrak{b}}$ definierte Funktor von der Form $\Omega(f)$ ist.

Bemerkung 3.2. Wenn $F$ über $\mathfrak{A}$ in $\mathscr{P}$ steht, dann ist in $\mathscr{P}$ sogar $\mathfrak{B}$ zu ${ }^{\mathfrak{I} \mathfrak{b}}$ isomorph.

Beweis. Sei $G: \mathfrak{B} \rightarrow{ }_{s} \mathfrak{U b}$ eine Äquivalenz von Kategorien und $f: R \rightarrow S$ ein $A$-Algebrenhomomorphismus. Dann ist $\Omega(f) G$ ein vergessender Funktor. Sei umgekehrt $F: \mathfrak{B} \rightarrow{ }_{R} \mathfrak{A} \mathfrak{b}$ ein vergessender Funktor. Dann ist $F \cong \operatorname{Hom}_{R}(R, F \cdot)$. Da $F$ einen links-adjungierten Funktor ${ }^{*} F$ besitzt, ist $\operatorname{Hom}_{R}(R, F \cdot) \cong \operatorname{Hom}_{\mathfrak{F}}\left({ }^{*} F R, \cdot\right)$, wobei ${ }^{*} F R$ durch die Multiplikation eine $R$-Rechts-Struktur besitzt. Da $F \cong \operatorname{Hom}_{\mathfrak{B}}\left({ }^{*} F R, \cdot\right)$ einen rechts-adjungierten Funktor besitzt, ist ${ }^{*} F R$ projektiv, und da $F$ eine Einbettung ist, ist ${ }^{*} F R$ ein projektiver Generator. Da ${ }^{*} F$ einen rechts-adjungierten Funktor besitzt, ist ${ }^{*} F$ mit direkten Summen vertauschbar, also existieren direkte Summen von ${ }^{*} F R$ in $\mathfrak{B}$. Weiter gilt für ${ }^{*} F R \cong{ }^{*} F R_{i}$ folgende Isomorphie:

$$
\sum \operatorname{Hom}_{\mathfrak{B}}\left({ }^{*} F R,{ }^{*} F R_{i}\right) \cong \sum F\left({ }^{*} F R_{i}\right) \cong F\left(\sum^{*} F R_{i}\right) \cong \operatorname{Hom}_{\mathfrak{B}}\left({ }^{*} F R, \sum^{*} F R_{i}\right) .
$$

Damit sind die Voraussetzungen für [2, S. 405, Cor. 1] gegeben und für $S=$ $\operatorname{Hom}_{\mathfrak{B}}\left({ }^{*} F R,{ }^{*} F R\right)$ definiert $\operatorname{Hom}_{\mathfrak{B}}\left({ }^{*} F R, \cdot\right)$ eine Äquivalenz zwischen $\mathfrak{B}$ und

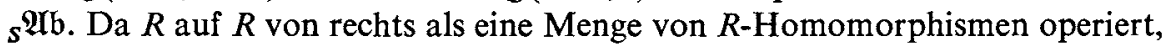
ist dadurch ein $A$-Algebrenhomomorphismus $f: R \rightarrow S$ definiert. Man zeigt damit, daß $\Omega(f) \operatorname{Hom}_{\mathfrak{B}}\left({ }^{*} F R, \cdot\right)$ isomorph zu $F$ ist.

Eine direkte Folge aus der Definition des vergessenden Funktors ist

Bemerkung 3.3. Die Hintereinanderausführung zweier vergessender Funktoren in $\mathscr{P}$ ist wieder ein vergessender Funktor. 
Bemerkung 3.4. Seien $F: \mathfrak{B} \rightarrow \mathfrak{C}$ und $G: \mathfrak{C} \rightarrow{ }_{R} \mathfrak{H b}$ Funktoren in $\mathscr{P}$ und seien $\mathfrak{B}, \mathfrak{C}$ abelsche Kategorien. Sind $G \cdot F$ und $G$ vergessende Funktoren, so ist auch $F$ ein vergessender Funktor.

Beweis. Nach Satz 3.1 ist $\mathfrak{B} \cong s_{s} \mathfrak{l b}$, da $G \cdot F$ vergessender Funktor ist, und $\mathfrak{C} \cong{ }_{T} \mathfrak{U b}$, da $G$ vergessender Funktor ist. Wir haben also ein Diagramm

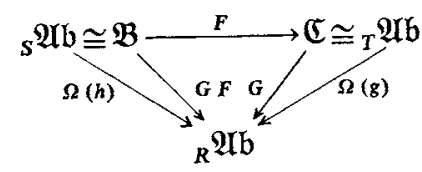

Da $R$ vermöge $k: A \rightarrow R$ eine $A$-Algebra ist, steht das ganze Diagramm durch $\Omega(k):{ }_{R} \mathfrak{A b} \rightarrow{ }_{A} \mathfrak{A b}$ über ${ }_{A} \mathfrak{I b}$. Speziell ist

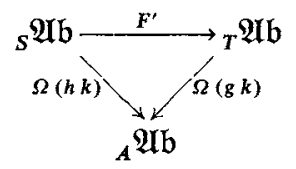

bis auf Isomorphie kommutativ. Das ist ein Diagramm in $\mathscr{\mathscr { P }}$ für $\mathfrak{A}={ }_{A} \mathfrak{M b}$ und $\left({ }_{s} \mathfrak{U b}, \Omega(h k)\right) \cong(\Omega S, V)$ bzw. $\left({ }_{T} \mathfrak{U b}, \Omega(g k)\right) \cong(\Omega T, V)$. Nach Satz 1.2 ist daher $F^{\prime} \cong \Omega(f)$ mit einem $A$-Algebrenhomomorphismus $f: T \rightarrow S$.

\section{Endomorphismenringe}

Seien $S$ eine $A$-Algebra, $\mathfrak{B}$ eine $A$-präadditive Kategorie und $F: \mathfrak{B} \rightarrow s^{\mathfrak{I} \mathfrak{b}}$ ein $A$-additiver Funktor. Dann können wir $F$ auffassen als Funktor über $\boldsymbol{A}^{\mathfrak{A} \mathfrak{b}}=\mathfrak{Q}$ durch das Diagramm

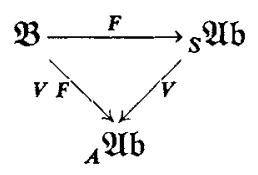

Im allgemeinen wird $F$ kein vergessender Funktor sein, also auch $V \cdot F$ nicht.

Wir wollen jetzt das folgende universelle Problem in $\mathscr{P}$ lösen: es stehe $\mathfrak{B}$ durch $V \cdot F$ über ${ }_{A} \mathfrak{U b}$. Gesucht sind eine abelsche Kategorie $\mathfrak{C}$, die mit einem vergessenden Funktor über ${ }_{A} \mathfrak{A b}$ steht, also äquivalent $\mathrm{zu}_{E} \mathfrak{U} \mathfrak{U}$ mit einer $A$-Algebra $E$ ist, und ein Funktor $G: \mathfrak{B} \rightarrow{ }_{E} \mathfrak{A b}$, so daß jeder Funktor über ${ }_{A} \mathfrak{A b}$ von $\mathfrak{B}$ in eine abelsche Kategorie, die durch einen vergessenden Funktor über ${ }_{A} \mathfrak{A b}$ steht, eindeutig bis auf Isomorphie über $G$ faktorisierbar ist.

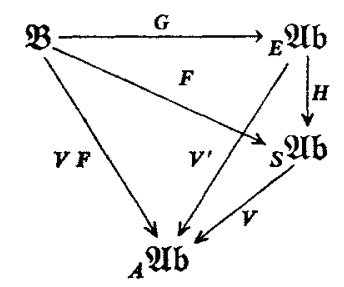


B. PAREIGIS:

Durch dieses universelle Problem wird der Teil des Funktors $F$, der ein vergessender Funktor ist, eindeutig abgetrennt.

Durch Satz 3.1 und Bemerkung 3.4 ist bewiesen, da B die volle Unterkategorie $\mathscr{A}_{\mathscr{Q}}$ von $\mathfrak{A P}_{\mathscr{P}}$ mit $\mathfrak{A}={ }_{A} \mathfrak{U} \mathfrak{b}$ bestehend aus den abelschen Kategorien, die mit vergessenden Funktoren über $\boldsymbol{A}_{\mathfrak{A b}}$ stehen, äquivalent zur Bildkategorie $\mathrm{Bi} \Omega$ von $\Omega: \mathscr{A}^{0} \rightarrow{ }^{\mathfrak{I}} \mathscr{P}$ ist. Wegen Satz 1.2 ist diese äquivalent $\mathrm{zu} \mathscr{A}^{0}$. Nach Definition ist der Funktor $\mathscr{A}^{0} \rightarrow \mathrm{Bi} \Omega \rightarrow{ }^{\mathfrak{Z}} \rightarrow{ }^{\mathfrak{I}} \mathscr{P}$ gerade der Funktor $\Omega$. Da $\Omega$ einen linksadjungierten Funktor $\Gamma$ besitzt, existiert auch $\mathrm{zu}^{\mathfrak{I} \mathscr{Q}} \rightarrow{ }^{\mathfrak{I}} \mathscr{P}$ ein links-adjungierter Funktor :

$$
(\mathfrak{B}, V F) \leadsto \Omega \Gamma(\mathfrak{B}, V F)=\Omega(\operatorname{End}(V F))=\Omega E .
$$

Satz 4.1. Sei $(\mathfrak{B}, H)$ ein Objekt in $\mathscr{P} \mathscr{P}$. Jede A-Algebra $S$ definiert einen vergessenden Funktor $V:{ }_{s} \mathfrak{U b} \rightarrow{ }_{A} \mathfrak{U} \mathfrak{b}$. Jeder Funktor $F: \mathfrak{B} \rightarrow{ }_{s} \mathfrak{U b}$ in ${ }^{\mathfrak{I} \mathscr{P}}$ läßt sich in eindeutiger Weise durch ${ }_{E} \mathfrak{H b}$ mit $E=\operatorname{End}(H)$ so faktorisieren, daß ${ }_{E} \mathfrak{A b} \rightarrow{ }_{s} \mathfrak{U b}$ ein vergessender Funktor ist. ${ }_{E}^{\mathfrak{U b}}$ ist bis auf Isomorphie in ${ }^{\mathfrak{P}}$ eindeutig durch diese Eigenschaft bestimmt.

Folgerung 4.2. Sei $F=\operatorname{Hom}_{R}(M, \cdot):{ }_{R} \mathfrak{U b} \rightarrow{ }_{s} \mathfrak{U b}$ gegeben, so läßt sich $F$ durch ${ }_{E} \mathfrak{A b}$ mit $E=\operatorname{End}_{R}(M)^{0}$ faktorisieren.

Folgerung 4.3. Sei $F=M \otimes_{R} \cdot:{ }_{R} \mathfrak{A b} \rightarrow{ }_{S} \mathfrak{A b}$ gegeben, so läßt sich $F$ durch ${ }_{E} \mathfrak{A b}$ mit $E=\operatorname{End}_{R}(M)$ faktorisieren.

Beweis. Durch Zusammensetzung mit dem vergessenden Funktor $V$ ist

bzw.

$$
\operatorname{End}(V F) \cong \operatorname{End}\left(\operatorname{Hom}_{R}(M, \cdot)\right) \cong \operatorname{End}_{R}(M)^{0}
$$

$$
\operatorname{End}(V F) \cong \operatorname{End}\left(M \otimes_{R} \cdot\right) \cong \operatorname{End}_{R}(M) .
$$

Also gilt Satz 4.1 mit $E=\operatorname{End}_{R}(M)^{0}$ bzw. $\operatorname{End}_{R}(M)$.

Wir wollen die Faktorisierungen von $F$ explizit angeben. Trivialerweise existiert ein $A$-Algebrenhomomorphismus $f: S \rightarrow E$. Also ist ${ }_{S} \mathrm{Hom}_{R}(M, \cdot) \cong$ $\Omega(f)_{E} \operatorname{Hom}_{R}(M, \cdot)$ und ${ }_{S} M \otimes_{R} \cong \Omega(f)_{E} M \otimes_{R} \cdot$.

Im folgenden wollen wir nur Funktoren zwischen Modulkategorien in $\mathscr{P}$ untersuchen. Seien $R, S A$-Algebren. Dann gilt

Lemma 4.4. Ein Funktor $F:{ }_{R} \mathfrak{A b} \rightarrow{ }_{S} \mathfrak{A b}$ besitzt dann und nur dann linksund rechts-adjungierte Funktoren, wenn er von der Form $\operatorname{Hom}_{R}(M, \cdot)$ für einen endlich erzeugten, projektiven $R-$ Modul $M$ ist.

Beweis. Man setze $M={ }^{*} F S$. Dann sieht man wie im Beweis von Satz 3.1, daß $M$ projektiv ist. Da $F$ als links-adjungierter Funktor von $F^{*}$ mit direkten Summen vertauschbar ist, ist $M$ endlich erzeugt. Umgekehrt gilt ${ }^{*} F \cong M \otimes_{S} \cdot$ und $F^{*} \cong \operatorname{Hom}_{S}\left(\operatorname{Hom}_{R}(M, R), \cdot\right)$, da nämlich die Funktoren $\operatorname{Hom}_{R}(M, \cdot)$ und $\operatorname{Hom}_{R}(M, R) \otimes_{R} \cdot$ isomorph sind, falls $M$ endlich erzeugt und projektiv ist.

Folgerung 4.5. Ein Modul $M_{R}$ ist genau dann endlich erzeugt und projektiv, wenn der Funktor $M \otimes_{R} \cdot:{ }_{R} \mathfrak{I b} \rightarrow{ }_{S} \mathfrak{H b}$ exakt und mit direkten Produkten vertauschbar ist. 
Beweis. Sei $M_{R}$ endlich erzeugt und projektiv. Dann ist $M \otimes_{R} \cdot \cong$ $\operatorname{Hom}_{R}\left(\operatorname{Hom}_{R}(M, R), \cdot\right)$ und hat einen links-adjungierten Funktor. $M \otimes_{\mathrm{R}} \cdot$ ist also mit projektiven Limites vertauschbar [1,3]. Sei umgekehrt $M \otimes_{R} \cdot$ mit projektiven Limites vertauschbar. Dann existiert [1, S. 89-90] ein linksadjungierter Funktor zu $F=M \otimes_{R^{*}}$, also ist nach Lemma $4.4 F \cong \operatorname{Hom}_{R}(N, \cdot)$ und $N$ ist endlich erzeugt und projektiv. Also ist auch $M \cong \operatorname{Hom}_{R}(N, R)$ endlich erzeugt und projektiv.

Man vergleiche hierzu auch die Definition eines platten Moduls.

Seien $R$ eine $A$-Algebra und $M, N R$-Moduln. Dann ist der Homomorphismus

$$
\varphi: \operatorname{Hom}_{R}(M, R) \otimes_{R} N \rightarrow \operatorname{Hom}_{R}(M, N)
$$

mit $\varphi(f \otimes n)(m)=f(m) n$ ein funktorieller Homomorphismus in $M$ und $N$. Ist $N=R$, so ist $\varphi$ ein Isomorphismus, also ist $\varphi$ ein Isomorphismus für endlich erzeugte, projektive Moduln $N$. Speziell gilt mit $E=\operatorname{Hom}_{R}(N, N)$ :

$$
{ }_{E} \operatorname{Hom}_{R}(N, R) \otimes_{R} N_{E} \cong{ }_{E} E_{E} .
$$

Lemma 4.6. Sei der $R$-Modul $N$ endlich erzeugt und projektiv, und sei $E \cong \operatorname{End}_{R}(N)^{0}$. Sei $F={ }_{E} \operatorname{Hom}_{R}(N, \cdot):{ }_{R} \mathfrak{A l b} \rightarrow{ }_{E} \mathfrak{A l b}$. Dann gilt $F \cdot{ }^{*} F \cong \operatorname{Id}\left({ }_{E} \mathfrak{I I b}\right)$.

Beweis. Es ist $F \cong_{E} \operatorname{Hom}_{R}(N, R) \otimes_{R} \cdot$ und $F \cdot{ }^{*} F \cong_{E} \operatorname{Hom}_{R}(N, R) \otimes_{R} N \otimes_{E} \cong$ ${ }_{E} E \otimes_{E} \cong \operatorname{Id}\left({ }_{E} \mathfrak{H b}\right)$.

Lemma 4.7. Sei der R-Rechts-Modul $N$ endlich erzeugt und projektiv, und sei $E \cong \operatorname{End}_{R}(N)$. Sei $F={ }_{E} N \otimes_{R} \cdot:{ }_{R} \mathfrak{A b} \rightarrow{ }_{E} \mathfrak{A l b}$. Dann gilt $F \cdot F^{*} \cong \operatorname{Id}\left({ }_{E} \mathfrak{H b}\right)$.

Beweis.

$$
\begin{aligned}
F \cdot F^{*} & \cong{ }_{E} N \otimes_{R} \operatorname{Hom}_{E}(N, \cdot) \cong \operatorname{Hom}_{R}\left(\operatorname{Hom}_{R}(N, R), \operatorname{Hom}_{E}(N, \cdot)\right) \\
& \cong \operatorname{Hom}_{E}\left(N \otimes_{R} \operatorname{Hom}_{R}(N, R), \cdot\right) \cong \operatorname{Hom}_{E}(E, \cdot) \cong \operatorname{Id}\left({ }_{E} \mathfrak{H b}\right)
\end{aligned}
$$

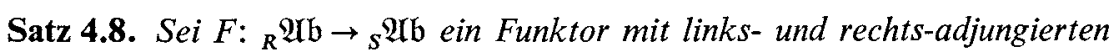
Funktoren. Sei $V:{ }_{S} \mathfrak{H} \mathfrak{b} \rightarrow{ }_{A} \mathfrak{A l b}$ der vergessende Funktor von $A \rightarrow S$. Sei $E=$ End $(V F)$. Dann gilt:

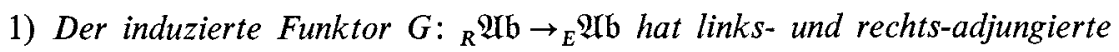
Funktoren.

2) $G \cdot G^{*} \cong G \cdot{ }^{*} G \cong \operatorname{Id}\left({ }_{E} \mathfrak{A b}\right)$.

Beweis. 1) folgt aus Lemma 4.4 und Folgerung 4.2. 2) folgt aus Lemma 4.6 und 4.7.

\section{Frobeniuserweiterungen}

Sei $A$ wieder ein kommutativer Ring und $\mathfrak{Q} \cong{ }_{A} \mathfrak{a t b}$. Eine spezielle Klasse von vergessenden Funktoren in ${ }^{\mathscr{M}} \mathscr{P}$ stellen die Funktoren dar, die durch den folgenden Satz charakterisiert werden.

Satz 5.1 [Morita]. Sei $f: S \rightarrow R$ ein A-Algebrenhomomorphismus. $f: S \rightarrow R$ ist dann und nur dann eine Frobeniuserweiterung, wenn ${ }^{*} \Omega(f) \cong \Omega(f)^{*}$ in $\mathscr{P}$. 
Zum Beweis siehe [5, Theorem 5.1]. Wir wollen daher allgemein einen Funktor $F$ einen Frobenius-Funktor nennen, wenn ${ }^{*} F$ und $F^{*}$ existieren und isomorph sind.

Bemerkung 5.2. Wenn $F$ ein Frobenius-Funktor ist, dann ist auch $F^{*}$ ein Frobenius-Funktor.

Wenn $F$ ein vergessender Frobenius-Funktor ist, ist nicht notwendig auch $F^{*}$ ein vergessender Frobenius-Funktor. Sei $F=\Omega(f):{ }_{R}^{\mathfrak{A b}} \rightarrow{ }_{s} \mathfrak{A b}$ ein vergessender Frobenius-Funktor. Dann haben wir für $F^{*}$ das Diagramm

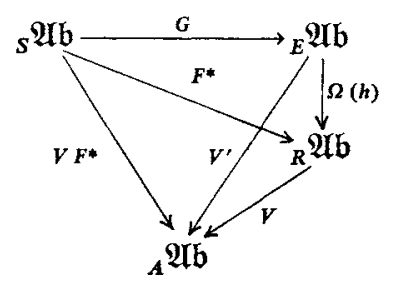

mit $E=\operatorname{End}\left(V F^{*}\right) \cong \operatorname{Hom}\left({ }_{s} R,{ }_{s} R\right)^{0}$ und $h: R \rightarrow E$, den $R$-Rechts-Multiplikatoren in $E$. Also ist $F^{*} \cong \Omega(h) G$. Da $F^{*}$ ein Frobenius-Funktor ist, ist ${ }^{*} G$. ${ }^{*} \Omega(h) \cong G^{*} \cdot \Omega(h)^{*}$ nach Satz 4.8.1 und weiter ${ }^{*} \Omega(h) \cong \Omega(h)^{*}$ nach Satz 4.8.2. Wegen Satz 5.1 ist also $h: R \rightarrow E$ eine Frobeniuserweiterung.

Folgerung 5.3 [KASCH]. Wenn $f: S \rightarrow R$ eine Frobeniuserweiterung ist, dann ist auch $h: R \rightarrow \operatorname{Hom}_{S}(R, R)^{0}$ eine Frobeniuserweiterung.

Eine Verallgemeinerung dieser Folgerung ist [5, Th. 9.3]. Wenn $G$ eine Äquivalenz von Kategorien ist, d.h. wenn $R$ ein $S$-Progenerator ist, gilt, wie man sofort sieht, auch die Umkehrung von Folgerung 5.3 [5, Th. 9.4].

Satz 5.4. Sei $f: S \rightarrow R$ eine Frobeniuserweiterung. Dann existiert ein Isomorphismus, so daß das folgende Diagramm kommutativ ist

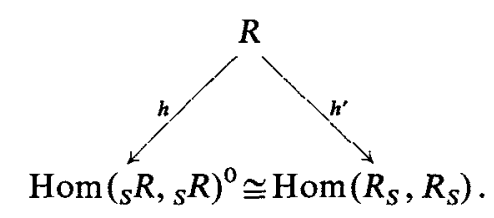

Beweis. Das Diagramm (*) bleibt kommutativ, wenn wir $F^{*}$ durch ${ }^{*} F$ ersetzen. Andererseits wird das universelle Problem durch ${ }_{E^{\prime}} \mathfrak{⿰ \mathfrak { A b }}$ mit $E^{\prime}=$ End $\left(V \cdot{ }^{*} F\right) \cong \operatorname{Hom}\left(R_{S}, R_{S}\right)$ gelöst. Also sind ${ }_{E}, \mathfrak{A b}$ und ${ }_{E} \mathfrak{H b}$ isomorph in ${ }^{\mathfrak{A}} \mathscr{P}$. Aber wir haben sogar das folgende Diagramm

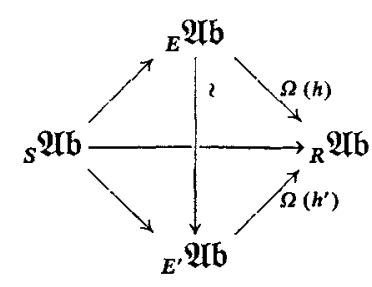


wobei alles zusätzlich über ${ }_{\mathcal{A}} \mathfrak{A} \mathfrak{b}$ steht. Der rechte Teil stellt ein Diagramm der Form

$$
\begin{gathered}
\Omega(E) \underset{\sim(h) \backslash / \Omega\left(h^{\prime}\right)}{\sim} \Omega\left(E^{\prime}\right) \\
\Omega(R)
\end{gathered}
$$

dar. Wegen Satz 1.2 ist daher die Behauptung bewiesen.

\section{Literatur}

[1] Freyd, P.: Abelian categories. New York: Harper and Row 1964.

[2] Gabriel, P.: Des catégories abéliennes. Bull. Soc. Math. France 90, 323-448 (1962).

[3] Kan, D. M.: Adjoint functors. Trans. Amer. Math. Soc. 87, 294- 323 (1958).

[4] KasCH, F.: Projektive Frobenius-Erweiterungen. Sitzungsber. Heidelb. Akad. Wiss., $89-109(1960 / 61)$.

[5] Morita, K.: Adjoint pairs of functors and Frobenius extensions. Sc. Rep. T.K.D. Sect. A. 9, 40-71 (1965).

[6] Séminaire Heidelberg-Strasbourg: Groupes algébriques. Exposé 1. Département Math. Strasbourg 1965.

Math. Inst. d. Univ. München, 8 München, Schellingstr. 2-8 